\title{
Epstein-Barr Virus and Breast Carcinoma in Iran
}

\author{
Maryam Kazemi Aghdam, ${ }^{1,2}$ Seyed Alireza Nadji, ${ }^{3}$ Maliheh Khoddami, ${ }^{1,2,}{ }^{*}$ Hoda Baghizadeh Dezfuli, \\ and Yassaman Khademi ${ }^{4}$ \\ ${ }^{1}$ Pediatric Pathology Research Center, Research Institute for Children Health, Shahid Beheshti University of Medical Sciences, Tehran, Iran \\ ${ }^{2}$ Department of Pathology, Medical School, Shahid Beheshti University of Medical Sciences, Tehran, Iran \\ ${ }^{3}$ Virology Research Center (VRC), National Research Institute of Tuberculosis and Lung Diseases (NRITLD), Shahid Beheshti University of Medical Sciences, Tehran, Iran \\ ${ }^{4}$ Pathologist, APCP, Pathobiology Laboratory Center, Tehran, Iran \\ "Corresponding author: Maliheh Khoddami, Pediatric Pathology Research Center, Research Institute for Children Health, Shahid Beheshti University of Medical Sciences, \\ Tehran, Iran. Tel: +98-2122227035, Fax: +98-2122227033, E-mail: malihehkhoddami@yahoo.com
}

Received 2017 May 08; Revised 2017 August 16; Accepted 2017 August 22.

\begin{abstract}
Background: A viral etiology for several malignancies has been suggested. One of the risk factors for development of breast carcinoma, which is the leading malignancy in women all over the world, is proposed to be a viral infection; hence recognition of the causative issues is essential for proper management.

Objectives: Epstein - Barr virus (EBV) infection is reported to be associated with breast carcinoma. This study was conducted to detect the presence of EBV-DNA in breast cancer in Iranian patients.

Methods: In this case-control study, the prevalence of EBV-DNA detection was investigated by qualitative real-time polymerase chain reaction (RT-PCR) using paraffin-embedded tissue samples of 75 women with breast cancer and 75 cases with benign breast lesions or normal breast tissue, which were examined between 2005 and 2014 at the pathobiology laboratory center, Tehran, Iran. The pathology reports of the patients were reviewed, retrospectively.

Results: In 7 cases $(9.3 \%$ ) of malignant lesions and $0 \%$ of benign lesions, the EBV-DNA was found, showing a statistically significant difference according to the Fisher test ( $\mathrm{P}=0.014$, Odds Ratio: 0.907; 95\% CI of OR: $0.843-0.975$ ).

Conclusions: According to our results, it can be suggested that EBV may have an etiologic role in breast cancer in Iran. The confirmation of the etiologic role of EBV in the induction of breast carcinoma needs more studies using more specific and sensitive techniques.
\end{abstract}

Keywords: Epstein - Barr Virus, Breast, Cancer, Polymerase Chain Reaction

\section{Background}

Although breast cancers are thought to be highly related to viruses, different researchers have presented controversial results (1). Breast cancer is a multi-stage disease and infection with DNA viruses may be contributing for some stages $(1,2)$. The DNA viruses may be oncogenic in many cancers such as Hepatitis B virus in hepatocellular carcinoma, Epstein-Barr virus (EBV) in lymphoma, nasopharyngeal cancer, and langerhans cell histiocytosis, as well as Human papilloma virus (HPV) in cervical cancer (1-3). Oncogenic viruses are contributing to $20 \%$ of human cancers. EBV, Human herpes virus type 8 (HHV-8), cytomegalovirus (CMV), HPV, and Herpes simplex virus-1 (HSV) were detected in breast malignant tissues by PCR (2).

Epstein-Barr virus from the Herpesviridae family has shown significant association with sporadic breast cancer cases and it has been suggested to be associated with the total survival rate (1) due to the presence of genes of this virus in breast tumoral tissues, however, not in normal tissues. Epstein-Barr virus may be not involved directly in breast carcinogenesis and may be the cause of cellular behavioral alteration to invasive patterns leading to the hypothesis that EBV is more common in breast cancers with negative receptors and higher grades (4). If convincing evidence for a carcinogenic role of these viruses in breast can be built up, there is a chance of primary prevention (4). Regarding controversies regarding the etiological role of EBV in development of breast cancer (5-11), this study was performed to detect the presence of EBV-DNA in breast tumors and show if there is any association of EBV virus and breast cancer in Iranian patients.

\section{Objectives}

The previous studies on this subject are limited in Iran. Early diagnosis and treatment of EBV, could be taken into consideration in the management of breast carcinoma. Therefore, we looked for EBV-DNA in the tumors of Iranian women diagnosed with breast cancer. 


\section{Methods}

\subsection{Ethics Statement}

Our patients were anonymous and the information extracted from the pathology reports is unchanged. The study protocol was approved by the Shahid Beheshti University of Medical Sciences ethic committee (SBMU.REC.1393.192).

\subsection{Patients and Controls}

The samples of 75 breast carcinomas used in this study were formalin fixed as well as paraffin-embedded and were diagnosed by the pathologist in 1 of the referral centers (pathobiology laboratory center) in Tehran, Iran, between 2005 and 2014. The criteria of diagnosis were taken from the related pathology textbooks. The slides were reexamined under the light microscope and adequate tumor samples were selected for the study. The criterion of exclusion was a small sized tumor. All cases were Iranian with a mean age of 48.2 years. For the control group, 75 non-tumoral tissue samples (reduction mastectomy, fibrocystic change (FCC), FCC and sclerosing adenosis, as well as FCC and focal adenomatoid change, ductal ectasia, and fat necrosis) were selected (2005 - 2014) from the files of the same department. Absence of malignancy was the criterion of inclusion.

\subsection{Tissue Preparation and DNA Extraction}

The embedding material should completely be removed from the $5 \mu \mathrm{m}$-thick-tissue samples prepared from paraffin-embedded blocks before DNA extraction. Xylene and alcohol were employed to deparaffinize the tissues and rehydrate, followed by tissue lysis using tissue lysis buffer and proteinase K. To extract DNA from lysed tissue, instructions of the company was followed (RTP ${ }^{\circledR}$ DNA/RNA Virus Mini Kit procedure; Stratec Molecular GmbH, Berlin, Germany) and the nucleic acids were frozen at $-20^{\circ} \mathrm{C}$.

\subsection{Polymerase Chain Reaction (PCR)}

The quality control of the extracted DNA was done by using the Sybr green real-time PCR-Melting curve for beta globin gene using GH20 primer; GAAGAGCCAAGGACAGGTAC and PCO4 primer; CAACTTCATCCACGTTCACC (Figure 1). The Thermo Scientific ${ }^{\mathrm{TM}}$ Maxima $^{\mathrm{TM}} \mathrm{SYBR}^{\mathrm{TM}}$ Green $2 \mathrm{X}$ qPCR master mix and 10 pmol per reaction of the $\mathrm{GH} 20 / \mathrm{PCO} 4$ primers were used to conduct the quality control (Figure 1). To preform RT- PCR, BamH1W EBV sequence primer sets was used. It amplifies 84 bp gene region of the EBV genome (ebv-f; 5'-GCAGCCGCCCAGTCTCT3'), ebv-r; 5'- ACAGACAGTGCACAGGAGCCT-3'). The internal probe was ebv-p; 5'-FAM- AAAAGCTGGCGCCCTTGCCTGBHQ1-3' (12). Reaction volumes of $20 \mu$ L underwent amplification as follows: 1) sample denaturation at $94^{\circ} \mathrm{C}$ for 10 minutes, 2) 10 second denaturation at $94^{\circ} \mathrm{C}$, 3) annealing and extension at $60^{\circ} \mathrm{C}$ ( 1 minute, 50 cycles) (Figure 2$)$. CFX96 RT-PCR System (BIO-Rad, USA) was employed and the reagent was HS prime taq premix taqman (GENETBIO, Korea). Serial dilutions of AmpliRun ${ }^{\circledR}$ EBVDNA CONTROL (Vircell, Spain) was used to detect the limit of 15 copies of EBV genome per reaction. The respective $\mathrm{r} 2$ values generated by the standard curves were 0.996 . The quality of the assays, in terms of efficiency, was $93.1 \%$.

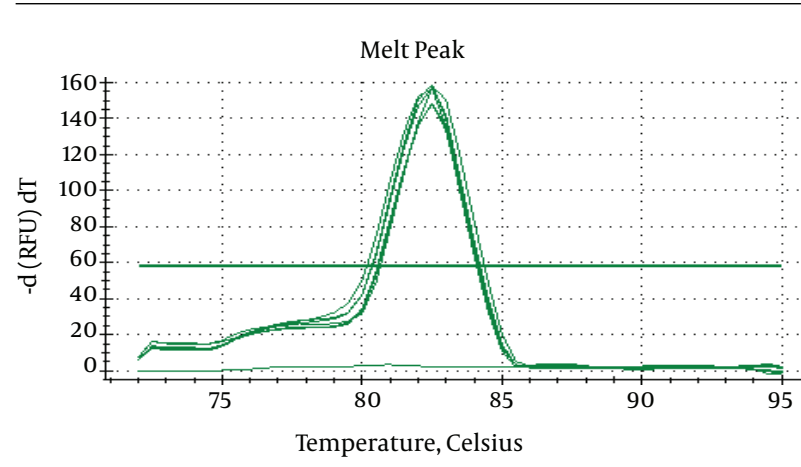

Figure 1. Temperature Around $82.5^{\circ} \mathrm{C}$ Demonstrated in Beta-Globin Gene SyberGreen RT-PCR-Melting Curve

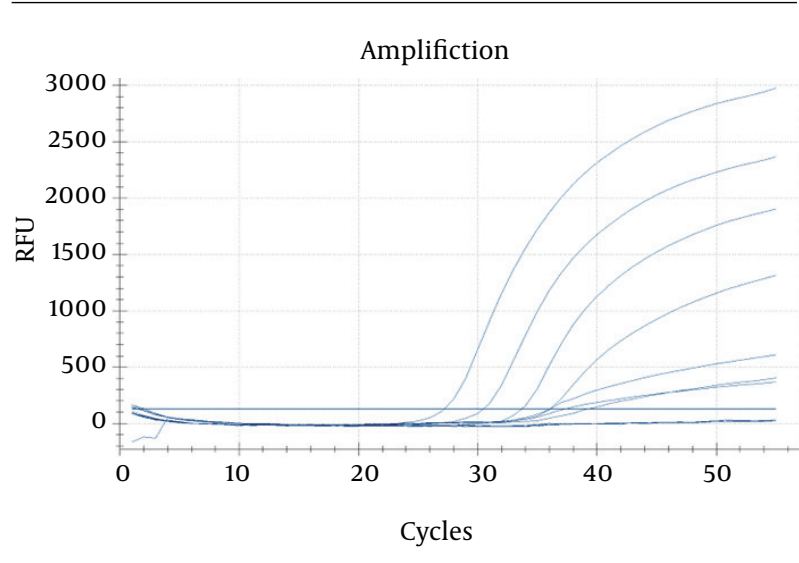

Figure 2. BamH1W Sequence Primer Sets Employed in EBV RT-PCR

\subsection{Statistical Analysis}

To perform the analysis, Chi-Square or Fisher's exact test was used. Statistical significance was a P value of less than 0.05 .

\section{Results}

The mean age in malignant cases was $48.2 \pm 10.8$ years. The malignant tumors were medullary carcinoma, tubular 
carcinoma, invasive lobular carcinoma (ILC), and invasive duct carcinoma (IDC) in $1.33 \%, 1.33 \%, 9.33 \%$, and $88 \%$, respectively. The malignant lesions were right-sided in $49.3 \%$ and left-sided in $50.7 \%$. The mean age in the control group was 38.9 years and $76 \%$ of lesions were right-sided. The control group included fibrocystic changes in $57.3 \%$, fatty breast tissue in $25.3 \%$, ductal ectasia in $1.3 \%$, and other types in $16 \%$ of cases. As shown in Table 1, the EBV was found in 7 cases (9.3\%) of malignant lesions and 0\% of benign lesions showing statistically significant difference according to Fisher test $(\mathrm{P}=0.014)$, the odds ratio was 0.907 (CI95\%: 0.843 0.975). Table 2 demonstrates EBV presence rate based on the type of lesions. Age, tumor laterality, and pathological type of the tumors were not related to the presence of EBV in malignant cases $(\mathrm{P}>0.05)$.

\section{Discussion}

Many researchers have been fascinated for decades by the hypothesis that a virus may cause human breast cancer. The link of hormone-responsive viruses with breast cancers cannot be excluded (HPV, mouse mammary tumor virus (MMTV) and EBV are the major candidates) and EBV is expressed in up to $50 \%$ of breast tumors by several researches $(1,4)$. In our study, EBV was detected in $9 \%$ of malignant lesions with no positivity in benign lesions or normal breast, showing statistically significant difference according to Fisher test.

Different studies have shown viruses in cells lines, in addition to their contribution to some breast tumors. The review article by Hsu et al. (1) reported that HPV, EBV, CMV, HSV-1, and HHV-8 may be contributing to breast cancer. The study by Lawson and colleagues (4) suggested the association between EBV and breast cancer. Tsai et al. (2) assessed 127 subjects in 2 groups of case and control and it was seen that HHV-8 and EBV were related to malignant status of lesions. Mazouni et al. (8) reported that 65 out of 196 breast cancer cases had EBV-DNA by RT-PCR, which was related to worse pathology subtype. Preciado and colleagues (11) showed presence of EBV in breast carcinoma with $35 \%$ positivity (using immunohistochemistry (IHC) in 69 breast carcinomas) as well as 31\% positive results (using PCR in 39 breast carcinomas) and zero rate for 48 control groups, however, there was no significant association between EBV expression and worse clinical or pathologic characteristics. They used the IHC method for EBV-encoded nuclear antigen 1(EBNA-1) and the positive results were approved with PCR.

In Mohamed's research (9), the EBV positive rate was $35.3 \%$ and they proposed a possible association between this virus and breast malignancy. Zekri et al. (13) assessed 90 Egyptian and Iraqi women with breast cancer and showed a 45\% (Egyptian) and 28\% (Iraqi) positive rate of EBV-DNA in malignant cases compared to none of benign cases, showing a significant difference. No statistically significant difference between EBV presence and tumor grade was reported in either population. The methods used included In situ hybridization (ISH) for EBV-specific RNA (EBER) and IHC for CD21 as well as PCR. The meta analysis performed by Huo and colleagues (6) in 2012, on researches using the PCR technique for detecting the EBV, including 24 studies and 1535 cases (1993 - 2008), showed EBV infection in $29.32 \%$ of the women with malignant breast tumors. The highest prevalence (35.25\%) was in Asian patients and the lowest (18.27\%) in Americans. Statistically, the strongest connection of EBV was with lobular carcinoma.

Glenn et al. (14) used the situ PCR method and reported that $68 \%$ and $35 \%$ of cases with breast cancer and control breastfeeding women had EBV-DNA. They also identified high risk HPV in 50\% and MMTV sequences in $78 \%$ of 50 invasive breast cancer specimens. More than 1 virus was detected in $72 \%$ of samples of the same breast carcinoma and in $13 \%$ of the same milk samples. They concluded that these viruses are probably related to a higher tumor grade and young age. The study by Richardson et al. (15), using both quantitative PCR and determination of serum immunoglobulin level for EBV and CMV, also reported significant difference between malignant and benign tissues in 70 subjects. Their review of the literature and the metaanalysis of all the results of PCR studies performed on breast carcinoma regarding CMV and EBV, raised the following possibilities: 1 , these analyses have limitations and cannot confirm whether the viruses are associated with breast cancer; however, using ISH in addition to PCR may increase the sensitivity; 2 , the virus may be absent after development of the tumor ('hit and run' oncogenesis), causing contradictory results; 3 , one or more viruses could be responsible for induction of breast carcinoma at a later period; 4, the possibility of breast carcinoma could be increased by infection with multiple viruses; 5 , none of the virus has a role in cancer development.

On the contrary to the positive reports, Perrigoue et al. (10) reported no significant association between EBV and breast cancer in 45 cases by RT- PCR and ISH. Joshi et al. (7), in their review of the literature in 2012, concluded that the data in the publications reviewed do not defend a conclusion that MMTV-like sequences, HPV, or EBV have an etiologic role in breast cancer. The 3 previous studies performed in Iran (Table 3) showed no significant positive results; Eghbali and colleagues (5) had a higher but statistically insignificant rate of HBV-DNA in malignant cases using the PCR technique. Kadivar et al. (16) reported that EBNA-1 and LMP-1 were negative in all 100 breast carcinoma cases and control subjects, which were approved by the 
Table 1. EBV Presence Rate in Malignant and Benign Lesions in Our Study

\begin{tabular}{lccc}
\hline Type & Positive & Negative & Total \\
\hline Malignant & $7(9.3 \%)$ & $68(90.7 \%)$ & $75(100 \%)$ \\
Benign & $0(0 \%)$ & $75(100 \%)$ & $75(100 \%)$ \\
Total & $7(4.7 \%)$ & $143(95.3 \%)$ & $150(100 \%)$ \\
\hline
\end{tabular}

Table 2. EBV Presence Rate Based on the Type of Lesions

\begin{tabular}{|c|c|c|c|c|c|}
\hline \multirow[t]{2}{*}{ Pathological Type } & & & \multicolumn{2}{|c|}{ EBV } & \multirow[t]{2}{*}{ Total } \\
\hline & & & Pos & Neg & \\
\hline \multirow{5}{*}{ Malignant } & \multirow{4}{*}{ Type } & IDC & $7(10.6)$ & $59(89.4)$ & $66(100.0)$ \\
\hline & & ILC & $0(0)$ & $7(100.0)$ & $7(100.0)$ \\
\hline & & Tubular & $0(0)$ & $1(100.0)$ & $1(100.0)$ \\
\hline & & Medullary carcinoma & $0(0)$ & $1(100.0)$ & $1(100.0)$ \\
\hline & Total & & $7(9.3)$ & $68(90.7)$ & $75(100.0)$ \\
\hline \multirow{5}{*}{ Benign } & \multirow{4}{*}{ Type } & Others & & $12(100.0)$ & $12(100.0)$ \\
\hline & & FCC & & $43(100.0)$ & $43(100.0)$ \\
\hline & & Fatty breast tissue & & $19(100.0)$ & $19(100.0)$ \\
\hline & & Ductal ectasia & & $1(100.0)$ & $1(100.0)$ \\
\hline & Total & & & $75(100.0)$ & $75(100.0)$ \\
\hline
\end{tabular}

Abbreviations: FCC, fibrocystic change; IDC, invasive ductal carcinoma; ILC, invasive lobular carcinoma.

PCR method. Also, the study by Fadavi et al. (17), using PCR and RT-PCR, showed negative EBV-DNA in 18 breast cancers. The false positive results may be due to contamination, infected circulating lymphocytes, cross-reaction of antibodies or use of ISH, which determines the virus with qualitative but not quantitative method. The negative results may be due to geographical differences and various distribution patterns of virus, low amount of the virus, and low sensitivity of used techniques (16). Also, some techniques may not distinguish between viruses in tumoral cells and those in tissue lymphocytes $(16,17)$.

\subsection{Conclusion}

According to our results and review of other studies in the literature, it can be concluded that EBV may have an etiologic role in breast cancer. No significant association between pathological subtype and EBV-DNA presence was identified in our study. Although the presence of a virus alone does not establish a causal role in the disease and other techniques are not used to prove the attendance of EBV- DNA in tumor cells, our findings with 9.3\% positivity, in addition to the previously reported positive results, supports the possibility of an etiologic relationship. The proof of etiologic role of EBV in the induction of breast carci- noma needs more efforts using more specific and sensitive techniques $(1,2,16,17)$. If the association of one or more of these viruses with breast cancer can be established, there is a possibility of prevention by anti-viral treatments and vaccination strategies (11). Limitations in our study included unavailability of IHC and ISH for EBV and absence of EBV serologic test results.

\section{Acknowledgments}

This research was performed as a thesis by Dr Hoda Baghizadeh Dezfuli under the direction of Dr Maliheh Khoddami and Dr Maryam Kazemi Aghdam, to fulfill the necessities of pathology speciality at Shahid Beheshti University of Medical Sciences. The study was supported by the pediatric pathology research center, Shahid Beheshti University of Medical Sciences, Tehran, Iran. We would like to thank Ali Tajic for his statistical analysis, as well as to Ms. Leila Poos-Ashkan and Pooneh Tavakoli who performed the PCR.

\section{Footnotes}

Authors' Contribution: Maryam Kazemi Aghdam: analysis and interpretation of data, revision of the manuscript, 
Table 3. Results of Previous Studies in Iran (Breast ca. and EBV)

\begin{tabular}{lcr}
\hline Study & Case Group & Control Group \\
\hline Eghbali et al (5) & $4.24(16.7 \%)$, no association & $1.24(4 \%)$ \\
Kadivar et al (16) & $0.100(0 \%)$ & $0.42(0 \%)$ \\
Fadavi et al (17) & $0.18(0 \%)$ & - \\
Current study & $7.75(9.3 \%)$ & IHC, PCR \\
PCR & $11.217(5.1 \%)$ & $0.75(0 \%)$ \\
\hline
\end{tabular}

and final approval of the version to be published; Seyed Alireza Nadji: supervising and performing PCR tests, analysis and interpretation of PCR data; Maliheh Khoddami: corresponding author, conception and design, supervision, critical revision of the manuscript; Hoda Baghizadeh Dezfuli: collecting data, drafting of the manuscript; Yassaman Khademi: review and diagnosis of the tumors and control cases, collecting data.

Financial Disclosure: This study is financially supported by the pediatric pathology research center, mofid children's hospital, Shahid Beheshti University of Medical Sciences.

\section{References}

1. Hsu CR, Lu TM, Chin LW, Yang CC. Possible DNA viral factors of human breast cancer. Cancers (Basel). 2010;2(2):498-512. doi: 10.3390/cancers2020498. [PubMed: 24281079].

2. Tsai JH, Tsai CH, Cheng MH, Lin SJ, Xu FL, Yang CC. Association of viral factors with non-familial breast cancer in Taiwan by comparison with non-cancerous, fibroadenoma, and thyroid tumor tissues. J Med Virol. 2005;75(2):276-81. doi: 10.1002/jmv.20267. [PubMed: 15602723].

3. Khoddami M, Nadji SA, Dehghanian P, Vahdatinia M, Shamshiri AR. Detection of Epstein-Barr Virus DNA in Langerhans Cell Histiocytosis. Jundishapur J Microbiol. 2015;8(12):27219. doi: 10.5812/jjm.27219. [PubMed: 26870310].

4. Lawson JS, Gunzburg WH, Whitaker NJ. Viruses and human breast cancer. Future Microbiol. 2006;1(1):33-51. doi: 10.2217/17460913.1.1.33. [PubMed: 17661684].

5. Eghbali M, Ghane M, Mirinarges M, Mehrjardi AZ. Detection of epstein barr virus among benign and malignant breast tumors. Asian J Biolog Sci. 2012;5(7):365-71. doi: 10.3923/ajbs.2012.365.371.

6. Huo Q, Zhang N, Yang Q. Epstein-Barr virus infection and sporadic breast cancer risk: a meta-analysis. PLoS One. 2012;7(2):31656. doi: 10.1371/journal.pone.0031656. [PubMed: 22363698].

7. Joshi D, Buehring GC. Are viruses associated with human breast cancer? Scrutinizing the molecular evidence. Breast Cancer Res Treat. 2012;135(1):1-15. doi: 10.1007/s10549-011-1921-4. [PubMed: 22274134].
8. Mazouni C, Fina F, Romain S, Ouafik L, Bonnier P, Brandone JM, et al. Epstein-Barr virus as a marker of biological aggressiveness in breast cancer. Br J Cancer. 2011;104(2):332-7. doi: 10.1038/sj.bjc.6606048. [PubMed: 21179039].

9. Mohamed WS, Mohamed MA, Omar MM. Possible involvement of Epstein-barr Virus (EBV) in pathogenesis and prognosis of female breast infiltrating duct carcinoma: clinicopathological, immunohistochemical and molecular study. The Egypt J Med Microbiol. 2007;16(2):403-14.

10. Perrigoue JG, den Boon JA, Friedl A, Newton MA, Ahlquist P, Sugden B. Lack of association between EBV and breast carcinoma. Cancer Epidemiol Biomarkers Prev. 2005;14(4):809-14. doi: 10.1158/1055-9965.EPI04-0763. [PubMed: 15824148].

11. Preciado MV, Chabay PA, De Matteo EN, Gonzalez P, Grinstein $\mathrm{S}$, Actis A, et al. Epstein-Barr virus in breast carcinoma in Argentina. Arch Pathol Lab Med. 2005;129(3):377-81. doi: 10.1043/15432165(2005)129<377:EVIBCI>2.0.CO;2. [PubMed: 15737034].

12. Fan H, Kim SC, Chima CO, Israel BF, Lawless KM, Eagan PA, et al. EpsteinBarr viral load as a marker of lymphoma in AIDS patients. J Med Virol. 2005;75(1):59-69. doi:10.1002/jmv.20238. [PubMed:15543571].

13. Zekri AR, Bahnassy AA, Mohamed WS, El-Kassem FA, El-Khalidi SJ, Hafez MM, et al. Epstein-Barr virus and breast cancer: epidemiological and molecular study on Egyptian and Iraqi women.J Egypt Natl Canc Inst. 2012;24(3):123-31. doi: 10.1016/j.jnci.2012.06.001. [PubMed: 22929918].

14. Glenn WK, Heng B, Delprado W, Iacopetta B, Whitaker NJ, Lawson JS. Epstein-Barr virus, human papillomavirus and mouse mammary tumour virus as multiple viruses in breast cancer. PLoS One. 2012;7(11):48788. doi: 10.1371/journal.pone.0048788. [PubMed: 23183846].

15. Richardson AK, Currie MJ, Robinson BA, Morrin H, Phung Y, Pearson $\mathrm{JF}$, et al. Cytomegalovirus and Epstein-Barr virus in breast cancer. PLoS One. 2015;10(2):0118989. doi: 10.1371/journal.pone.0118989. [PubMed: 25723522].

16. Kadivar M, Monabati A, Joulaee A, Hosseini N. Epstein-Barr virus and breast cancer: lack of evidence for an association in Iranian women. Pathol Oncol Res. 2011;17(3):489-92. doi: 10.1007/s12253-0109325-z. [PubMed: 21207256].

17. Fadavi P, Rostamian M, Arashkia A, Shafaghi B, Niknam HM. Epsteinbarr virus may not be associated with breast cancer in Iranian patients. Oncol Discov. 2013;1(3) doi: http://dx.doi.org/10.7243/2052-61991-3. 